Am Ende des Lebens ist der Tod - wir wissen es alle und denken in der Regel möglichst wenig daran. Dass die Medizin den Tod hinauszuzögern vermag wie noch nie und vielen von uns ein gesundes Alter ermöglicht, manchen aber auch Monate oder sogar Jahre eines Schwebezustandes zwischen Leben und Tod bringt, lässt heute viele Menschen darüber nachdenken, was sie von ihrem Arzt in einer kritischen Situation erwarten. Eine Patientenverfügung kann eine Chance sein, dem Thema - auch im Gespräch zwischen Ärzten und ihren Patienten - Raum zu geben.

Dr. med. Christine Romann,

Mitglied des Zentralvorstands der FMH,

Verantwortliche Ressort Gesundheitsförderung und Prävention

\title{
Neue Patientenverfügung der FMH und der SAMW
}

Recht auf Selbstbestimmung - Niemand ist davor sicher: Durch einen Unfall oder eine Krankheit ist man nicht mehr in der Lage, selbständig Wünsche zu äussern und Entscheidungen $\mathrm{zu}$ treffen. Immer mehr Menschen sorgen deshalb mit einer Patientenverfügung vor und legen fest, welchen medizinischen Massnahmen sie im Falle ihrer Urteilsunfähigkeit zustimmen und welchen nicht. Im Zuge der Revision des Schweizerischen Zivilgesetzbuches ZGB unterstreicht der Bund das Recht auf Selbstbestimmung schweizweit: Ärztinnen und Ärzte haben einer Patientenverfügung zu entsprechen, sofern diese nicht gegen gesetzliche Vorschriften verstösst und keine begründeten Zweifel in Bezug auf den freien und mutmasslichen Willen der Patientin oder des Patienten bestehen. Das revidierte Erwachsenenschutzrecht, welches 2013 in Kraft tritt, wirkt sich damit auch auf das Arzt-Patienten-Verhältnis aus.

\section{Urteilsunfähigkeit bei Unfall oder Krankheit: Eine Patientenverfügung bietet Ärzten und weiteren medizinischen Fachpersonen Orientierungshilfe.}

Das vielgepriesene Recht auf Selbstbestimmung beinhaltet aber auch die Freiheit eines jeden Menschen, keine Patientenverfügung zu verfassen. Dies scheint ob all der möglichen Vorteile gelegentlich in Vergessenheit zu geraten. Druckversuche irgendwelcher Art, sei es von Angehörigen, Institutionen oder der öffentlichen Meinung, sind entschieden abzulehnen, zumal solche Erwartungshaltungen vor dem Hintergrund nie enden wollender Spardiskussionen und Kostenexplosionsszenarien bei bestimmten Bevölkerungsgruppen nachvollziehbare Ängste bewirken können. Die Patientenverfügung ist im neuen Erwachsenenschutzrecht des ZGB geregelt - sie darf nicht als Mittel zur Kosteneindämmung des KVG instrumentalisiert werden!

Ausführliche Version und Kurzversion - Patientenverfügungen entsprechen einem grossen Bedürfnis: Auf der Website der FMH gehört die Mustervorlage zu den meist aufgerufenen Dokumenten. Die grosse Nachfrage nach der FMH-Patientenverfügung und die Änderung des Schweizerischen Zivilgesetzbuches sowie das inzwischen schier unüber- schaubare Angebot an (zum teil kostenpflichtigen und tendenziösen) Vorlagen haben die FMH und die Schweizerische Akademie der Medizinischen Wissenschaften SAMW bewogen, die bisherige FMH-Patientenverfügung gemeinsam zu überarbeiten. Zur Verfügung stehen neu zwei Varianten, welche sich an den von der SAMW erarbeiteten Richtlinien orientieren: eine Kurzversion in Anlehnung an das bekannte Muster und eine ausführlichere Version, welche Anregungen und Raum für spezifische Inhalte gibt. Nützliche Hinweise zum Ausfüllen der Patientenverfügung finden sich in den

\section{Patientenverfügung in zwei Varianten - einfach downloaden auf www.fmh.ch.}

Erläuterungen. Mit diesem Angebot wird unterschiedlichen Bedürfnissen Rechnung getragen. So kann die Patientenverfügung einerseits so knapp wie möglich und andererseits so ausführlich wie nötig abgefasst werden, um Interpretationsschwierigkeiten zu vermeiden. Falls sich Antworten nicht direkt aus der Verfügung entnehmen lassen, kann die Beschreibung von Werthaltung und Motivation des Patienten eine zuverlässige Interpretationshilfe bieten.

Eine Patientenverfügung kann dazu einladen, über wichtige Fragen zu reden - Mit einer Patientenverfügung können Patient und Arzt wichtige Fragen frühzeitig klären und sich gemeinsam auf schwierige Situationen vorbereiten: Der Patient überlegt sich, welche Behandlungen er wünscht oder ablehnt, bespricht seine Anliegen und Befürchtungen mit seinem Arzt und hält seinen Willen in der Patientenverfügung schriftlich fest. Dies kann eine grosse Entlastung für die Angehörigen sein, da sie nicht mehr um die Frage ringen müssen, was der Patient wohl gewollt hätte. Zu wünschen ist, dass die Patientenverfügung primär auch zum Gedankenaustausch zwischen Angehörigen und mit Vertrauenspersonen anregt und für solche Gespräche bedenkenswerte Fragen und Inhalte vorschlägt - selbst wenn vorerst keine Verfügung unterschrieben wird. Die Patientenverfügung sowie eingehendere Informationen finden Sie auf www.fmh.ch.

Lucia Rabia, Fürsprecherin, stv. Leiterin Rechtsdienst FMH 